\title{
Theorie des diskursiven Institutionalismus
}

Im Folgenden werden zunächst die Grundgedanken des diskursiven Institutionalismus dargelegt und danach ein Überblick über die zentralen Konzepte dieser Theorieströmung gegeben: Definitionen und Kategorien von Ideen, von Akteur*innen als Träger dieser Ideen, von Diskursen sowie Institutionen. Im darauffolgenden Schritt werden Arten von Politikwandel dargestellt - diese reichen von graduellem zu transformativem Wandel - sowie deren Voraussetzungen. Abschnitt 3.4 gibt einen Überblick, welche Studien bisher den diskursiven Institutionalismus verwendet haben. Im letzten Abschnitt dieses Kapitels wird dann ein Zwischenfazit gezogen, in dem die in dieser Arbeit verwendeten Kategorien und Begriffe herausgestellt und miteinander in Verbindung gebracht werden.

\subsection{Grundgedanken des diskursiven Institutionalismus}

Der diskursive Institutionalismus liefert einen Erklärungsansatz, wie sich Politik durch Ideen und Diskurse verändern kann. Er reiht sich damit in weitere Strömungen des Neoinstitutionalismus ein: den rational-choice-Institutionalismus, den historischen Institutionalismus und den soziologischen Institutionalismus, die unterschiedliche Erklärungen dafür bieten, wie Institutionen politische Entscheidungen beeinflussen, wie sie sich verändern und wie Institutionen zu definieren $\operatorname{sind}^{1}$.

\footnotetext{
${ }^{1}$ Einige Autor*innen (u. a. Schimank 2007) zählen weitere Theorieansätze dazu, wie die Institutionenökonomik und den akteurszentrierten Institutionalismus. An dieser Stelle beziehe ich mich aber auf die meistverbreitete Kategorisierung in drei Hauptströmungen des Neoinstitutionalismus und die Neuerung des diskursiven Institutionalismus.
} 
Laut rational-choice-Institutionalismus treffen Akteur*innen rationale und kalkulierte Entscheidungen und folgen dabei ihren persönlichen Interessen. Der historische Institutionalismus geht dagegen davon aus, dass politische Institutionen als routinierte Praktiken und regulierte Strukturen Handlungen leiten und betont die Einschränkung von Entscheidungen durch Pfadabhängigkeit. Veränderungen sind daher nur schwierig umzusetzen und geschehen weitestgehend durch Druck von außen, durch den sich sogenannte „windows of opportunities“ beziehungsweise Gelegenheitsfenster auftun. Laut Capoccia und Kelemen (2007, S. 305) sind diese Ereignisse von kurzer Dauer im Vergleich zu den langen Phasen der Stabilität und ermöglichen es, neue Wege in der Politik einzuschlagen. Die dritte Strömung, der soziologische Institutionalismus, lenkt den Blick auf soziale Akteur*innen, die nach erlernten Handlungsmustern agieren und Erwartungssicherheit anstreben. Laut dieser Strömung ermöglichen Institutionen es, die Handlungen von anderen Akteur*innen einzuschätzen und das eigene Handeln daran zu orientieren (Schimank 2007, S. 164-170; Schmidt 2010, S. 2).

Campbell beschreibt Forderungen nach einem „Second Movement“ im Institutionalismus seit den 1990er Jahren, bei dem die verschiedenen Strömungen zusammengebracht und sinnstiftend ergänzt werden (Campbell 2004, S. 4). Im Zuge dessen haben sich seit einigen Jahren vermehrt Autor*innen dieser verschiedenen Strömungen des Neoinstitutionalismus auch der Bedeutung von Ideen und Diskursen sowie Veränderungen gewidmet, die nicht auf Vorkommnisse von außen zurückzuführen sind. Sie brechen dabei teilweise mit den Traditionen ihrer neoinstitutionalistischen Schule, weshalb Schmidt diese als neue Strömung bezeichnet und sie diskursiven Institutionalismus nennt (Schmidt 2010, S. 2). Andere Autor*innen fassen diese Ansätze unter dem Namen konstruktivistischer Institutionalismus zusammen (u. a. Hay 2006, S. 57). In den vorliegenden Buch wird der häufiger verwendete Begriff des diskursiven Institutionalismus genutzt. Die meisten Überschneidungen gibt es dabei mit dem soziologischen Institutionalismus; aber auch aus dem rational-choice Institutionalismus und dem historischen Institutionalismus kommende Autor*innen können dem diskursiven Institutionalismus zugeordnet werden (Schmidt 2002, S. 8, 2010, S. 9-13). Bei diesen Autor*innen spielen jeweils außer Ideen und Diskursen auch noch weitere Aspekte wie rationale Entscheidungen, exogene Schocks und Veränderungen oder Machtgefüge ebenfalls eine Rolle (Schmidt 2010, S. 21). Daher ist nicht immer klar abzugrenzen, welche Ansätze sich zwar Diskursen widmen, aber noch in einer der drei älteren Strömungen verbleiben und welche dem diskursiven Institutionalismus zugerechnet werden können. Schmidt (2008, S. 304) empfiehlt, die Autor*innen dazu zu zählen, die in ihren Aussagen den Annahmen der anderen Institutionalismusansätze widersprechen. 
Der diskursive Institutionalismus untersucht Diskurse und Ideen, um Veränderungen und Kontinuität von Politik zu erklären. Er beschäftigt sich außerdem mit dem institutionellen Kontext, in dem und durch den Ideen kommuniziert werden (Schmidt 2008, S. 314, 2010, S. 2-4). Im Gegensatz zu den anderen Neoinstitutionalismusansätzen wird dabei die Handlungsfähigkeit von Akteur*innen betont, die einerseits durch ihre Umgebung sozialisiert sind, andererseits aber auch strategisch handeln können (Hay 2006, S. 58; Schmidt 2008, S. 316). Aufbauend auf dieser Annahme wird beim diskursiven Institutionalismus davon ausgegangen, dass Veränderungen nicht immer nur als Reaktion auf exogene Schocks und andere Vorkommnisse zu verstehen sind, sondern auch durch interne Prozesse und Entscheidungen von Akteur*innen zu erklären sind (Hay 2006, S. 65; Schmidt 2010, S. 1).

Eine Analyse dieser Diskursprozesse ermöglicht zu erklären, warum einige Ideen einflussreich werden und andere nicht, je nachdem, wie sie in einem bestimmten Kontext von welchen Akteur*innen kommuniziert werden (Schmidt 2008, S. 309). Diese Theorieströmung eignet sich dazu, herauszufinden, wie sich neue Ideen, so auch die von Wissenschaftler*innen, verbreiten und wann sie erfolgreich Politik beeinflussen können.

\subsection{Zentrale Konzepte des diskursiven Institutionalismus}

\subsubsection{Ideen}

Die Autor*innen, die dem diskursiven Institutionalismus zugeordnet werden, unterscheiden verschiedene Kategorien von Ideen mit unterschiedlichen Funktionen in Diskursen. An dieser Stelle werden unterschiedliche Kategorisierungen von Schmidt (2008, 2017) und Campbell (2004) genannt und miteinander in Verbindung gebracht (siehe Tab. 3.1).

Schmidt (2008, S. 305) und Campbell (2004, S. 93) unterscheiden beide zwischen kognitiven Ideen - was vorhanden und was zu tun ist, um ein bestimmtes Ergebnis zu erreichen - und normativen Ideen - was gut und was schlecht ist und wie gehandelt werden sollte. Kognitive Ideen sind Richtlinien für politische Handlungen, die es erleichtern, politische Entscheidungen zu rechtfertigen, indem auf Interessen und Notwendigkeiten verwiesen wird (Schmidt 2008, S. 306). Sie sind auch Erklärungen über Ursache-Wirkungs-Zusammenhänge zwischen mehreren Variablen (Campbell 2004, S. 93). Ideen dieses Typs sind nur dann erfolgreich, wenn sie logisch, relevant und passend für den Kontext erscheinen. Dabei können 
sie von wissenschaftlichen Erkenntnissen unterstützt werden, wobei der Wahrheitsgehalt nicht für den Erfolg einer kognitiven Idee entscheidend ist (Schmidt 2006, S. 251). Normative Ideen sind verknüpft mit Werten, die politische Entscheidungen legitimieren, indem sie auf deren Angemessenheit verweisen (Schmidt 2008, S. 307). Erfolgreich können sie also nur dann sein, wenn sie mit bestimmten Werten und Normen übereinstimmen, was sowohl neu aufkommende Werte als auch bestehende sein können (Schmidt 2006, S. 252).

Gleichzeitig unterscheidet Schmidt $(2008,2017)$ zwischen drei Ebenen von Ideen, die unterschiedlich leicht veränderbar sind: policy ideas, programmatic ideas und philosophical ideas. Erstere sind konkrete politische Entscheidungen, die sich häufig verändern, insbesondere, wenn die bisher angewandte Politik die Probleme nicht mehr zu lösen scheint. Programmatic ideas verändern sich hingegen seltener, und zwar zumeist in Zeiten hoher Unsicherheit. Sie beinhalten Ideen über Methoden, Instrumente und Ziele von Politik und verbinden diese zu einem umfassenden Programm. Die dritte Art von Ideen, philosophical ideas, bettet die politischen Entscheidungen und Programme in eine größere Idee ein und verändert sich nur sehr selten. Sie beinhalten beispielsweise Weltansichten oder Ideologien (Schmidt 2017, S. 251). Klenk und Larson (2015, S. 21) beziehen sich auf die Unterscheidung Schmidts und konkretisieren sie bezüglich einer zeitlichen Komponente, indem sie argumentieren, dass policy und programmatic ideas eher unmittelbar gedacht sind, während philosophical ideas die längerfristige Richtung zeigen. Programmatic ideas verstehen sie außerdem als in enger Verbindung zu Problemen und deren Lösungen stehend.

Andere Autor*innen nehmen abweichende Unterscheidungen zwischen den Arten von Ideen vor. So unterscheidet Campbell (2004, S. 93) zusätzlich zu normativen und kognitiven Ideen noch zwischen Ideen, die im Hintergrund liegen und Ideen, die im Vordergrund präsent sind und artikuliert werden (siehe Tab. 3.1). Während sich die Ideen im Vordergrund leicht und häufig ändern, sind die Ideen im Hintergrund langlebiger und hemmen Veränderungen (Campbell 2004, S. 93). Eine Kombination der Unterscheidungen zwischen normativen und kognitiven Ideen sowie im Hintergrund und im Vordergrund liegenden Ideen ergibt vier Arten von Ideen: Paradigmen, öffentliche Empfindungen, Politikprogramme und Rahmen (Campbell 2002, S. 21, 2004, S. 93).

Paradigmen sind laut Campbell (2004, S. 94 f.) kognitive Annahmen, die im Hintergrund liegen und die Auswahl an Alternativen, die von Entscheidungsträger*innen als nützlich angesehen werden, einschränken. Dies sind beispielsweise Annahmen darüber, wie Familienstrukturen aufgebaut sind, was Einfluss darauf hat, welche Unterstützungen der Wohlfahrtsstaat bereitstellen sollte. Paradigmen stecken ein Spektrum ab, innerhalb dessen Politik gemacht und Entscheidungen 
Tab.3.1 Kategorisierung von Ideen

\begin{tabular}{l|l|l|l|l}
\hline & \multicolumn{2}{|l|}{ Nach Campbell (2004) } & \multicolumn{2}{l}{ Nach Schmidt (2008, 2017) } \\
\hline & Kognitiv & Normativ & Kognitiv/normativ & \\
\hline Hintergrund & Paradigmen & Empfindungen & Philosophical ideas & 3. Ebene \\
\hline \multirow{2}{*}{ Vordergrund } & Programme & - & Programmatic ideas & 2. Ebene \\
\cline { 2 - 5 } & - & Frames & Policy ideas & 1. Ebene \\
\hline
\end{tabular}

Die Tabelle zeigt verschiedene Kategorien von Ideen und deren Verortung im Hinter- und Vordergrund der Diskurse. Von Ebene eins bis drei, beziehungsweise vom Vorder- zum Hintergrund steigt der Schwierigkeitsgrad der Veränderung.

Quelle: Campbell (2004, S. 93) und Schmidt (2008, S. 305, 2017, S. 251). Eigene Darstellung.

getroffen werden. Somit beeinflussen Paradigmen, was als möglich angesehen wird (Campbell 2002, S. 22 f.): „[T]aken-for-granted paradigms constrain the range of policies that policy makers are likely to consider". In früheren Artikeln betonen bereits andere Autor*innen, die dem diskursiven Institutionalismus zugeordnet werden können (u. a. Hall 1993, S. 279; Hay 2001, S. 200), die Bedeutung von Politikparadigmen. Hall (1993, S. 279) führte diesen Begriff in die Strömung des Neoinstitutionalismus ein und argumentierte, dass Politiker*innen vor dem Hintergrund eines Sets an Ideen und Standards - den Politikparadigmen - handeln, welche beeinflussen, was als gegeben angesehen und nicht hinterfragt wird. Auch Kjaer und Pedersen (2001, S. 224) argumentieren, dass Paradigmen, ähnlich wie formale Institutionen, den politischen Diskurs strukturieren. Diese Art von Ideen ändert sich nur selten und geht mit Pfadabhängigkeiten einher. Sie entspricht in den Grundzügen den philosophical ideas nach Schmidt (2008).

Als zweite Art von Ideen nennt Campbell öffentliche Empfindungen. Diese liegen ebenfalls im Hintergrund, sind normative Annahmen darüber, was richtig und falsch ist. Dies sind Werte, Normen, Identitäten und geteilte Erwartungen, beispielsweise darüber, wofür ein Staat zuständig ist und wofür nicht, was wiederum politische Entscheidungen beeinflusst (Campbell 2004, S. 96 f.). Außerdem beeinflussen sie, wie Akteur*innen ihre Rollen und Interessen wahrnehmen. Sie schränken daher mögliche Entscheidungen ein, was als akzeptabel oder legitim angesehen wird. Die öffentlichen Empfindungen entsprechen keiner der drei von Schmidt genannten Kategorien im Detail. Da sie aber wie die Paradigmen im Hintergrund angesiedelt sind und Wandel eher hemmen, können sie den philosophical ideas zugeordnet werden. 
Programme sind Theorien oder Konzepte, die den Entscheidungsträger*innen als Vorschläge dienen, wie ein bestimmtes Problem gelöst werden kann, beispielsweise Sozialprogramme, die konkrete Ziele und Schritte vorgeben. Dadurch beeinflussen und erleichtern sie die Entscheidungsfindung und den Politikwandel. Programme sind kognitive Ideen, die im Vordergrund des politischen Diskurses angesiedelt sind, also bewusst zugänglich und relativ leicht veränderbar sind, im Gegensatz zu den oft unbewussten Paradigmen und öffentlichen Empfindungen (Campbell 2004, S. 98). Programme entsprechen den von Schmidt (2008) genannten programmatic ideas.

Auch Frames sind im Vordergrund des politischen Diskurses angesiedelt, werden aber normativ begründet. Diese Art von Ideen erlaubt es Eliten, ihre Programme und institutionellen Veränderungen bewusst mithilfe von Symbolen und Konzepten zu legitimieren. So können politische Veränderungen beispielsweise mit Bezug auf den Frame der traditionellen Familienstruktur legitimiert werden. Frames können einerseits Politikwandel verhindern, können ihn aber auch erleichtern, je nachdem, wie sie eingesetzt werden. Sie spielen insbesondere dann eine Rolle, wenn Ideen und politische Entscheidungen der Öffentlichkeit präsentiert und legitimiert werden (Campbell 2004, S. 98-100). Da sie bewusst genutzt werden und leicht veränderbar sind, werden sie hier auf der Ebene der policy ideas eingeordnet, haben jedoch keine genaue Entsprechung bei Schmidts (2008) Kategorisierung.

Die zwei Kategorisierungen lassen sich also wie folgt zusammenführen (siehe auch Tab. 3.1): Auf der untersten Ebene befinden sich Policies (Schmidt 2017, S. 251) und Frames (Campbell 1998, S. 99 f.). Sie liegen im Vordergrund des politischen Diskurses und sind leicht veränderbar. Dabei können Frames als normativ und Policies als kognitiv beziehungsweise outcome-orientiert verstanden werden. In Campbells Unterscheidung fehlen die Policies, die hier jedoch einbezogen werden sollen. Warum Campbell diese außen vorgelassen hat, wird nicht deutlich. Auf der zweiten Ebene stehen Programme, die sich auch regelmäßig ändern, was jedoch mit größeren Hürden verbunden ist als bei der ersten Ebene. Auf der dritten Ebene, den philosophical ideas, sind letztlich Paradigmen und öffentliche Empfindungen, die beide im Hintergrund stehen, sich nur sehr selten ändern und Politikwandel eher hemmen.

\subsubsection{Akteur*innen}

Wie bereits erläutert nehmen Akteur*innen laut diskursivem Institutionalismus eine entscheidende Rolle bei Politikveränderungen ein, weil sie Ideen entwickeln 
und verbreiten. Den im vorherigen Abschnitt genannten Arten von Ideen ordnet Campbell dabei anhand ihrer Funktionen bestimmte Akteur*innengruppen zu. Er unterscheidet dabei Entscheidungsträger*innen, Theoretiker*innen, Framer*innen und Auftraggeber*innen sowie Vermittler*innen (Campbell 2004, S. 100 f.).

Entscheidungsträger*innen - wie Politiker*innen und Verwaltungsmitarbeitende - wählen Programme aus oder entwickeln sie selbst. Wegen ihrer weitreichenden Entscheidungsbefugnis sind sie wichtige Akteur*innen in Bezug auf politischen Wandel. Die Entwicklung und Umsetzung von Programmen liegt hauptsächlich bei ihnen, wobei auch sie in ihrer Wahrnehmung und bei ihren Entscheidungen von verinnerlichten Paradigmen und öffentlichen Empfindungen beschränkt werden (Campbell 2004, S. 94-102).

Theoretiker*innen wie Wissenschaftler*innen oder andere Intellektuelle unterstützen und verbreiten einzelne Paradigmen; sie entwickeln und propagieren teilweise auch neue Paradigmen. Außerdem leiten sie daraus neue Programme her oder unterstützen bestehende (Campbell 2004, S. 102). Laut Skowronek (1982, S. 31) gibt es in allen stark institutionalisierten Staaten Gruppen von Intellektuellen, die über einen längeren Zeitraum enge Verbindungen zur Politik pflegen und mit Entscheidungsträger*innen Ideen austauschen.

Framer*innen beschreiben Programme passend zu bestehenden öffentlichen Empfindungen, damit diese als legitim angesehen werden und nutzen dafür bewusst geeignete Frames. Zu dieser Gruppe gehören beispielsweise Kampagnenmanager*innen und Medienberater*innen (Campbell 2004, S. 103).

Als weitere Gruppe sieht Campbell Auftraggeber*innen bzw. Wähler*innen (engl. Original: constituents), zu denen er die allgemeine Öffentlichkeit zählt. Je nachdem, wen eine politische Entscheidung betrifft, können jedoch auch Entscheidungsträger*innen selbst oder andere Gruppen Auftraggebende sein. An diese Gruppe richten sich die Framer*innen, wenn sie Programme rechtfertigen und sich dabei auf die verbreiteten öffentlichen Empfindungen beziehen (Campbell 2004, S. 104).

Die fünfte und letzte Gruppe von Akteur*innen, die Vermittler*innen, stehen zwischen den vorher genannten Gruppen und zwischen verschiedenen Arten von Ideen. Sie transportieren Ideen von einem Bereich in einen anderen. $\mathrm{Zu}$ ihnen zählen Berater*innen, Intellektuelle und Medien. Beispielsweise durch Policy Briefs bringen sie etwa Paradigmen oder Politikprogramme von der Forschung zur Politik und verbreiten ihre Ideen in der Presse, um so öffentliche Empfindungen zu beeinflussen (Campbell 2004, S. 104-107). Oft bilden Vermittler*innen epistemische Gemeinschaften (,epistemic communities“) - Netzwerke von Wissenschaftler*innen, Expert*innen oder NGOs zu bestimmten Themen - die neue Paradigmen und Programme entwickeln und diese an Entscheidungsträger*innen 
herantragen. Laut Haas (1992, S. 3 f., 27-29) werden diese epistemischen Gemeinschaften insbesondere dann um Rat gefragt, wenn hohe Unsicherheiten bestehen, beispielsweise zum Umgang mit Gefahren. Je mehr sich eine epistemische Gemeinschaft in einem politischen Kontext etabliert hat, umso mehr kann sie ihren Einfluss institutionalisieren. In der Folge werden ihre Ideen dann vermehrt in Politik und Gesellschaft akzeptiert.

Hier zeigt sich also eine Überschneidung zwischen den Theoretiker*innen und Vermittler*innen, die beide in der Wissenschaft verankert sein können, beide Paradigmen entwickeln und Programme daraus ableiten. Den Vermittler*innen spricht Campbell (2004, S. 104-107) die Rolle zu, die Ideen in unterschiedliche Bereiche zu verbreiten, wobei er insbesondere die Verbreitung von Programmen erwähnt, jedoch auch Paradigmen und andere Arten von Ideen:

„Particularly since the early 1970s, think tanks in the United States and Europe have become increasingly interested in simplifying and disseminating the paradigms and programs of other researchers rather than conducting research themselves. They have learned to transmit these ideas to decision makers through pithy policy briefs designed to affect programmatic thinking." (Campbell 2004, S. 106)

Campbell (2004, S. 177 f.) nennt außerdem die Kategorie der institutionellen Unternehmer*innen, deren Vorhandensein Voraussetzung für Politikwandel ist, da diese neue Ideen voranbringen. Diese können aus den unterschiedlichen, vorher genannten Gruppen, kommen. Um ihre Ideen umzusetzen, müssen sie außerdem über finanzielle, politische und organisationale Ressourcen verfügen (Campbell 2004, S. 178). Campbell bezieht sich mit dem Begriff institutioneller Unternehmer*innen auf DiMaggio (1988, S. 14), der damit Akteur*innen beschreibt, die über Ressourcen verfügen und ein Interesse an institutioneller Veränderung haben, da sie in der Veränderung eine Möglichkeit sehen, ihre Interessen zu realisieren. Lawrence und Suddaby (2006, S. 217) haben diesen Begriff aufgegriffen jedoch argumentiert, dass es zusätzlich zu den institutionellen Unternehmer*innen weiterer Akteur*innen bedarf, die deren Arbeit unterstützen. Diese Unterstützungsaktivitäten verschiedener Akteur*innen haben sie analysiert und kategorisiert (Lawrence und Suddaby 2006, S. 220-229). Im Rahmen der Schaffung neuer Institutionen beobachten sie, dass Akteur*innen konkrete neue Regeln und Rechte einführen oder dafür werben. Als zweite Kategorie bezeichnen sie Aktivitäten, bei denen Glaubenssysteme, Identitäten, Normen und um diese herum gespannte Netzwerke verändert und konstruiert werden. Die dritte Art von Aktivitäten bezieht sich auf die abstrakteren Konzepte, wobei einerseits neue Ideen entwickelt werden und andererseits Methoden vermittelt werden, diese 
umzusetzen (Lawrence und Suddaby 2006, S. 220 f.). Diese unterschiedlichen Aktivitäten können zum Teil direkt den Akteur*innengruppen von Campbell zugeordnet werden. So wird hier angenommen, dass insbesondere Theoretiker*innen neue abstrakte Ideen entwickeln und insbesondere die Framer*innen Normen, Glaubenssysteme und Identitäten konstruieren.

In Gesellschaften existieren immer verschiedene Werte gleichzeitig, teilweise widersprechen sie sich, teilweise gelten sie nur in bestimmten Kontexten oder zu bestimmten Zeiten und sie verändern sich kontinuierlich. Wenn Politiker*innen sich in ihren Diskursen auf bestimmte Werte beziehen, ist dies immer ein selektiver Prozess, der strategisch geführt werden kann (Schmidt 2005, S. 232). Diese und andere rhetorische Strategien sind Machtinstrumente, die politische Eliten nutzen können, um Entscheidungen zu beeinflussen. Ihre Position garantiert den Politiker*innen, mit ihren Argumenten gehört zu werden und Diskurse mitzugestalten, was alleine jedoch nicht für Veränderung ausreicht (Grube und van Acker 2017, S. 195).

Zusammengefasst zeigt sich, dass die genannten Gruppen von Akteur*innen einige Überschneidungen zeigen, beispielsweise zwischen Theoretiker*innen und Vermittler*innen, zu denen jeweils epistemische Gemeinschaften gezählt werden. Jeder Gruppe werden bestimmte Arten von Ideen zugeordnet, die insbesondere von diesen verändert und vorangebracht werden. So entwickeln Entscheidungsträger*innen insbesondere Programme, während Theoretiker*innen Paradigmen verändern und vertreten können. Framer*innen legitimieren Programme mithilfe von Frames und adressieren damit insbesondere die Auftraggeber*innen mit ihren öffentlichen Empfindungen. Vermittler*innen nutzen Ideen aller Kategorien und bringen diese von einem Kontext in einen anderen (Campbell 2004, S. 101-105). Wie Paradigmen von einer Gruppe zur anderen kommen und ob sich lediglich die Theoretiker*innen und Vermittler*innen diese bewusstmachen und die anderen Akteur*innen nur die abgeleiteten Programme wahrnehmen, bleibt unklar. Außerdem fällt auf, dass auch hier keine Policies erwähnt werden. Abgeleitet von der in Abschnitt 3.2.1 genannten Unterscheidung wird hier davon ausgegangen, dass Theoretiker*innen neben Programmen auch Policies ableiten und verbreiten und Entscheidungsträger*innen und Vermittler*innen neben Programmen auch Policies entwickeln. Dementsprechend legitimieren Framer*innen nicht nur Programme, sondern auch Policies gegenüber den Auftraggeber*innen. 


\subsubsection{Diskurse}

Schmidt (2002, S. 210) definiert Diskurse als interaktive Prozesse, in denen Ideen kommuniziert und ausgehandelt werden. Sie würden bestehen aus „whatever policy actors say to one another and to the public in their efforts to generate and legitimate a policy programme" (Schmidt 2002, S. 210). Da hier die Ideen mit dem Kontext, in dem sie formuliert werden, verschwimmen, werden in dieser Arbeit weitere Definitionen von Diskursen herangezogen und die Definition von Diskursen nach Schmidt (2002, S. 210) dementsprechend konkretisiert. Foucault (2015, S. 156) definiert Diskurse als ,eine Menge von Aussagen, die einem gleichen Formationssystem zugehören“. Kjaer und Petersen (2001, S. 220), Vertreter des diskursiven Institutionalismus, beschreiben Diskurse als

,a system of meaning that orders the production of conceptions and interpretations of the social world in a particular context. In this view, ideas are always embedded in discourses and become meaningful only by being interpreted as part of a particular discursive system of meaning“.

Schlussfolgernd werden Diskurse hier als die interaktiven Kontexte verstanden, in denen Ideen von bestimmten Akteur*innen dargelegt werden. Ideen, also Paradigmen, öffentliche Empfindungen, Policies, Programme und Frames sind dagegen die Inhalte dieser Diskurse, wobei insbesondere die drei letztgenannten offen formuliert werden.

Abgeleitet von der Unterscheidung kognitiver und normativer Ideen sieht Schmidt (2002, S. 213) zwei Funktionen von Ideen im Diskurs ${ }^{2}$ : Die kognitive Funktion, bei der Politikprogramme gerechtfertigt werden, indem Vorteile bestimmter Lösungen kommuniziert werden und die normative Funktion, die bei der Legitimierung von Politikprogrammen die Angemessenheit in einem bestimmten Kontext und eine Verbindung zu bestehenden Werten aufzeigt.

So werden Ideen ihrer kognitiven Funktion in Diskursen nur gerecht, wenn sie relevant und die kommunizierten Informationen glaubwürdig erscheinen. Die empfohlenen Aktionen müssen außerdem als machbar und sinnvoll wahrgenommen werden und zeigen, wie sie die empfundenen Probleme lösen werden (Schmidt 2002, S. 217-219). Werden Ideen unterschiedlicher Ebenen (siehe Abschn. 3.2.1) im Diskurs miteinander verknüpft, müssen diese stimmig sein, die Konzepte, Normen, Methoden und Instrumente eines Politikprogrammes also

\footnotetext{
${ }^{2}$ Schmidt (2002, S. 213) bezeichnet diese als Funktionen von Diskursen. Aufgrund der eben erwähnten konkreteren Definition von Diskursen in dieser Arbeit werden diese Funktionen hier auf die Ideen innerhalb von Diskursen bezogen.
} 
erklärt werden, ohne deutliche Widersprüche zu den genannten Policies oder Paradigmen zu offenbaren (Schmidt 2002, S. 219). Darüber hinaus muss eine Idee, um erfolgreich zu sein, im Vergleich zu anderen Politikprogrammen dargestellt werden, um zu zeigen, dass sie Probleme besser lösen kann, als die anderen möglichen Programme (Schmidt 2002, S. 219).

Darüber hinaus müssen die Ideen im Diskurs normative Kriterien erfüllen, um erfolgreich zu sein. So muss ein vorgeschlagenes Programm in Bezug zu bestehenden Normen und Werten gesetzt werden und zeigen, dass es diesen entspricht (Schmidt 2002, S. 219). Allerdings müssen Diskurse sich nicht immer auf bestehende Werte berufen. Fordert ein Diskurs eine radikale Transformation von bestehenden Werten, so kann er sich auch auf neu aufkommende Werte berufen und damit einen Wertewandel bestärken (Schmidt 2002, S. 221). Auch wenn Diskurse die Ideen verständlich und handlungsanleitend hervorbringen müssen, kann in einigen Fällen gerade auch eine Ungenauigkeit und Mehrdeutigkeit einem Diskurs zum Erfolg verhelfen, da sich Akteur*innen mit unterschiedlich ausgeprägten normativen Ideen ihm zuordnen können (Schmidt 2006, S. 253).

Schmidt unterscheidet zwei Arten von Diskursen (2002, S. 210, 2008, S. 303 , 2010, S. 16): Auf der einen Seite den koordinativen Diskurs, bei dem politische Akteur*innen untereinander kommunizieren, Programme entwickeln und politische Entscheidungen in der Öffentlichkeit präsentieren, begründen und rechtfertigen. Akteur*innen sind sowohl Politiker*innen und Verwaltungsmitarbeitende, die die politischen Entscheidungen koordinieren, als auch Medien, Aktivist*innen, Intellektuelle, Expert*innen, Think-tanks, Interessengruppen, epistemische Gemeinschaften und soziale Bewegungen (Schmidt 2006, S. 253, 2008, S. 310). Hier spielen insbesondere die kognitiven Kriterien von Ideen eine Rolle (Schmidt 2002, S. 221).

Auf der anderen Seite findet der kommunikative Diskurs zwischen politischen Akteur*innen und der Öffentlichkeit statt und kommuniziert die im koordinativen Diskurs entwickelten Ideen wie Politikprogramme, vor allem in Bezug auf die normativen Kriterien der Ideen. Akteur*innen sind öffentliche Verwaltungsmitarbeitende, gewählte Vertreter*innen, Expert*innen, Interessengruppen und Aktivist*innen, die sich in politische Entscheidungen einbringen wollen sowie die allgemeine Öffentlichkeit (Schmidt 2006, S. 255, 2008, S. 310). Oft geht ein diskursiver Prozess von den politischen Eliten zur Öffentlichkeit, kann aber auch andersherum geschehen, von Aktivist*innen zur politischen Ebene, oder auch nur im Bereich der Zivilgesellschaft bleiben ohne die politische Ebene zu erreichen (Schmidt 2008, S. 311).

Diffundieren Ideen von einem Kontext in einen anderen - räumlich oder thematisch gesehen - müssen sie für den jeweiligen neuen Kontext als passend 
übersetzt werden (Campbell 2004, S. 65). Diffusion wird dabei von Campbell (2004, S. 77) verstanden als ,spread of institutional principles or practices with little modification through a population of actors“. Je größer die Unterstützung für die neue Idee ist, umso wahrscheinlicher ist eine Übersetzung der Idee während der Diffusion ohne große Abwandlungen (Campbell 2004, S. 81).

Für die vorliegende Arbeit kann aus den vergangenen Absätzen geschlussfolgert werden, dass Ideen im Diskurs sowohl normativen als auch kognitiven Kriterien gerecht werden müssen. Welches Kriterium im Fokus ist, liegt am jeweiligen Diskurs, in dem die Ideen vorgebracht werden und den daran beteiligten Akteur*innen. Daneben müssen Ideen übersetzt werden, wenn sie von einem Kontext in einen anderen diffundieren. Je besser sie zu den dortigen Gegebenheiten passen, umso weniger werden sie im Laufe der Übersetzung abgewandelt.

\subsubsection{Institutionen}

Institutionen werden im diskursiven Institutionalismus als Regeln oder Strukturen verstanden, die das Handeln von Individuen und Gruppen leiten. Sie können sowohl formelle Gesetze als auch informelle Regeln sein. Campbell (2004, S. 1) definiert sie als

,the foundation of social life. They consist of formal and informal rules, monitoring and enforcement mechanisms, and systems of meaning that define the context within which individuals, corporations, labor unions, nation-states, and other organizations operate and interact with each other.“

Hier werden Überschneidungen zwischen Institutionen und den im Hintergrund liegenden Ideen deutlich, da auch Paradigmen als „systems of meaning“ bezeichnet werden könnten und das Handeln von Akteur*innen beeinflussen. Im soziologischen Institutionalismus werden Institutionen definiert als ,letztlich jede Art von (dauerhaft) reproduzierten sozialen Praktiken, die sich in der Empirie für eine Organisation als bedeutungsvoll herausgestellt haben" (Senge und Hellmann 2006, S. 18). Auch Normen, Orientierungspunkte für Handeln, Werte und unbewusste Handlungsroutinen sind Institutionen, da sie das Handeln von Organisationen bestimmen (Senge und Hellmann 2006, S. 18). An Hay (2001, S. 213) orientiert stellt dagegen ein Paradigmenwechsel nicht automatisch institutionellen Wandel dar. Die Übersetzung eines Paradigmas in Institutionen ist ein längerer Prozess, der nicht immer erfolgreich ist. 
Campbell (2004, S. 1) weist darauf hin, dass die Institutionen stets die Ressourcen und Macht derjenigen reflektieren, die sie geschaffen haben und dadurch gleichzeitig die weitere Macht- und Ressourcenverteilung in einer Gesellschaft beeinflussen. Diese Institutionen beeinflussen, welche Ideen sich durchsetzen können und stellen daher wichtige Rahmenbedingungen dar. Sind neue Ideen einmal institutionalisiert, hemmen sie zukünftigen institutionellen Wandel und sorgen für Pfadabhängigkeit (Campbell 2004, S. 110). Dadurch und durch ihren Einfluss auf das Handeln von Menschen und wie sie ihre Umwelt wahrnehmen, tragen sie zu einer Stabilisierung der sozialen Welt bei (Campbell 2004, S. 1).

Durchlässige Institutionen ermöglichen es institutionellen Unternehmer*innen im Vergleich zu sehr isolierten Institutionen, sich einzubringen und Veränderungen anzustoßen (Campbell 2004, S. 178). Wie genau sich durchlässige Institutionen auszeichnen, wird jedoch bei Campbell (2004) nicht deutlich. Laut Sheingate (2003, S. 193) können institutionelle Unternehmer*innen Innovationen besonders gut dann einbringen, wenn Unsicherheiten oder Unklarheiten über institutionelle Strukturen bestehen. Dies kann durch externe Vorkommnisse geschehen, aber beispielsweise auch in Zeiten von Wahlkämpfen.

\subsection{Politikwandel laut diskursivem Institutionalismus}

\subsubsection{Arten und Intensitäten von Politikwandel}

Die Vertreter*innen des diskursiven Institutionalismus beschreiben unterschiedliche Intensitäten von Wandel, die mit der Veränderung von Ideen auf unterschiedlichen Ebenen einhergehen. Auch wenn im diskursiven Institutionalismus und anderen Strängen des Neoinstitutionalismus meist von Veränderungen von Institutionen gesprochen wird, geht diesen stets eine Veränderung von vorherrschenden Ideen sowie von politischen Entscheidungen und Policies voraus. Hier wird sich zunächst dem Politikwandel in seinen unterschiedlichen Ausprägungen zugewandt.

Verändern sich die Dimensionen einer Institution nach und nach und nur geringfügig und passen sich den neuen Gegebenheiten an, sprechen Vertreter*innen des diskursiven Institutionalismus (u. a. Blyth 2002; Campbell 2004; Quack 2006) von graduellem oder inkrementellem Wandel. Wenn sich alle Dimensionen eines Diskurses abrupt verändern, sprechen sie von revolutionärem oder transformativem Wandel und beziehen sich auf den Begriff der „Great Transformation" von Polanyi (1944). 
Viele Autor*innen (u. a. Béland 2005; Blyth 2002; Cashore und Howlett 2007; Gardner 2017; Hay 2001; Schmidt 2008) verwenden dabei die von Hall (1993) eingeführte Klassifikation von Politikwandel. Er argumentiert, dass Politik aus drei zentralen Variablen besteht: Den Zielen der Politik, den Techniken oder Politikinstrumenten, die verwendet werden, um die entsprechenden Ziele zu erreichen sowie der spezifischen Ausgestaltung der Politikinstrumente (Hall 1993, S. 278). Politikwandel kann daraus abgeleitet von der geringfügigen Anpassung der Ausgestaltung der Instrumente (wie der Rentenhöhe, Wandel erster Ordnung), über eine Anpassung der Instrumente selbst (wie ein neues Rentensystem, Wandel zweiter Ordnung) bis hin zur Veränderung der Ziele dieser Politik (wie hinter der Altersvorsorge stehende Werte, Wandel dritter Ordnung) reichen. Während die Veränderungen erster und zweiter Ordnung einen gewöhnlichen Teil von Politik darstellen, ist die dritte Form der Veränderung eine radikale Transformation (Hall 1993, S. 278-281). Veränderungen erster und zweiter Ordnung geschehen regelmäßig, wenn Unzufriedenheit mit den bestehenden Instrumenten und vergangenen Erfahrungen aufkommt und die gesetzten Ziele nicht mehr zufriedenstellend erreicht werden (Hall 1993, S. 278-283). Veränderung dritter Ordnung passiert dagegen seltener und geht zudem mit einer Veränderung oder Erneuerung der bestehenden Paradigmen einher (Hall 1993, S. 279).

In diesem Zusammenhang argumentiert Hall, dass die Wahl zwischen konkurrierenden Paradigmen meist nicht nur auf Basis wissenschaftlicher Erkenntnisse getroffen werden kann und der Wechsel von einem vorherrschenden Paradigma zu einem neuen meist nicht nur von den Argumenten der Verfechter*innen des neuen Paradigmas abhängt, sondern auch von Macht- und Ressourcenverteilungen und äußeren Einflüssen (Hall 1993, S. 280). In demokratischen Gesellschaften findet der Kampf um konkurrierende Paradigmen und ein möglicher Paradigmenwechsel unter anderem in der Öffentlichkeit statt und beinhaltet Diskussionen und Abwägungen alternativer Paradigmen auch in den Medien (Campbell 2004, S. 34; Hall 1993, S. 286; Hay 2001, S. 200). Schmidt (2002, S. 223 f.) fügt dem jedoch hinzu, dass es keinen kompletten Austausch eines Paradigmas benötigt, um von Paradigmenwechsel zu sprechen, da stets unterschiedliche Paradigmen in einer Gesellschaft nebeneinander existieren. Ein Paradigmenwechsel ist also der Austausch des vorherrschenden Paradigmas, das politische Entscheidungen leitet, jedoch keine komplette Verdrängung oder Veränderung des früheren Paradigmas.

Laut Hay (2001, S. 213) kann transformativer Wandel beziehungsweise Paradigmenwechsel mit Institutionenwandel einhergehen, was aber nicht immer der Fall sein muss. Doch wenn es dazu kommt, kann die durchgesetzte Idee unabhängig von den Akteur*innen ein Eigenleben entwickeln und strukturiert damit Handeln und sorgt für Pfadabhängigkeiten (Berman 1998, S. 26 f.). Um diese 
unterschiedlichen Grade der Veränderung besser unterscheiden zu können, werden diese in diesem Buch weiter ausdifferenziert und anhand der Ebenen von Ideen (siehe Abschn. 3.2.1) zusammengeführt (siehe Abb. 3.1).

Stabilität $\quad$ Gradueller Wandel Transformativer Wandel

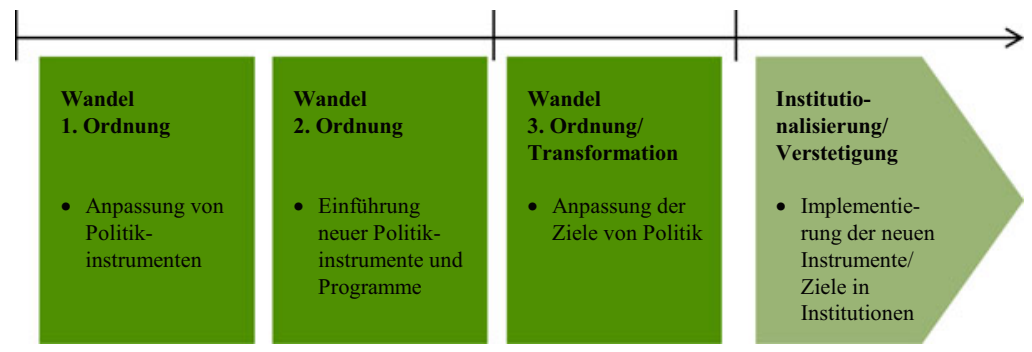

Abb. 3.1 Formen und Intensitäten von Politikwandel. Die Abbildung zeigt die Grade von Politikwandel. Während Stabilität eine Zeit ohne Veränderung ist, werden beim graduellen Wandel nur Politikinstrumente bzw. Policies angepasst (Wandel erster Ordnung) oder auch neue Instrumente oder Programme eingeführt (Wandel zweiter Ordnung). Bei Transformation werden auch die Ziele von Politik und die dahinterstehenden Paradigmen und öffentlichen Empfindungen verändert (Wandel dritter Ordnung). Auf alle Grade des Wandels kann eine Implementierung der neuen Instrumente oder Ziele in neue Institutionen folgen, was jedoch nicht immer der Fall ist.

Quelle: Hall (1993), Blyth (2002), Campbell (2004) und Hay (2001). Eigene Darstellung

Stabilität beschreibt den Zustand ohne Veränderung. Inkrementeller oder gradueller Politikwandel ist eine Veränderung von Ideen auf den Ebenen der Policies und Programme. Eine erste Stufe hiervon ist Veränderung erster Ordnung, bei der lediglich die Politikinstrumente angepasst werden beziehungsweise neue Policies umgesetzt werden. Eine zweite Stufe graduellen beziehungsweise inkrementellen Wandels, der Wandel zweiter Ordnung, beinhaltet neben neuen Policies auch die Einführung neuer Programme, jedoch noch keinen grundlegenden Wandel. Eine weitere Stufe von Veränderung ist die Transformation, ein grundlegender Wandel dritter Ordnung, der die Veränderung der Ziele von Politik sowie der Paradigmen beinhaltet. An letzter Stelle dieser Abstufung unterschiedlicher Formen von Politikwandel steht eine Institutionalisierung, bei der Policies, Programme oder Paradigmen verändert werden und es zusätzlich zu einer formellen Überführung 
der neuen Ideen in Institutionen kommt. Dadurch erhalten die neuen Ideen zusätzliche Stabilität und sind schwieriger zu verändern. Institutionalisierung kann sich aber sowohl auf die Verstetigung von kleinen Policies als auch von umfassenden Programmen oder gar Paradigmen beziehen, also unterschiedliche Intensitäten haben. Ausschlaggebend ist hier die Langfristigkeit der Veränderung.

\subsubsection{Voraussetzungen für Politikwandel}

In den folgenden Abschnitten werden Thesen aus der Theorie des diskursiven Institutionalismus zu der Frage herausgearbeitet, unter welchen Voraussetzungen es zu Politikwandel kommt und wann die Chance für höhere Grade von Wandel besteht. Dabei wird sich insbesondere an von Campbell (2004, S. 174181) genannten Thesen orientiert, diese jedoch mit den Annahmen weiterer Autor*innen zusammengeführt und anhand der drei Bereiche Krise, neue Idee und institutionelle Unternehmer*innen sortiert (siehe Abb. 3.2).
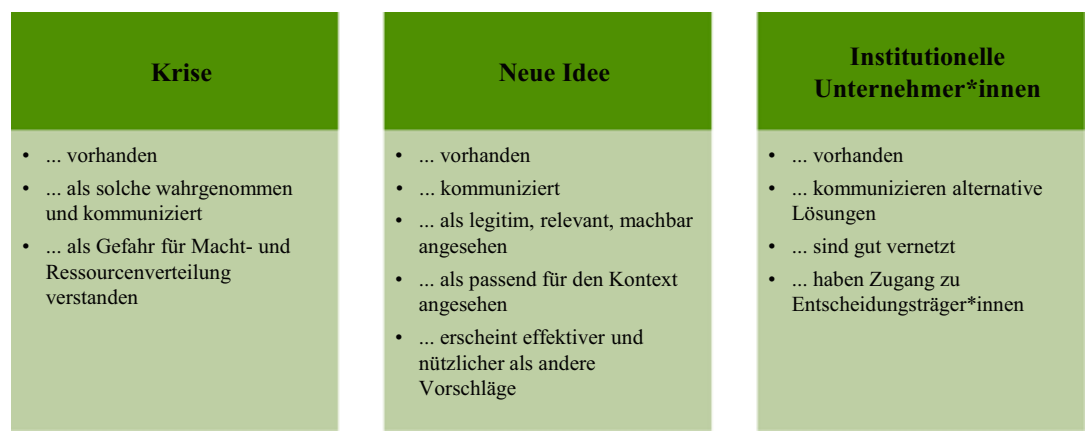

Abb. 3.2 Kriterien für Politikwandel. Die Abbildung zeigt Voraussetzungen für Politikwandel. Demnach muss eine Krise vorhanden sein, die von den Entscheidungsträger*innen als solche wahrgenommen und kommuniziert wird und als Gefahr für die Macht- und Ressourcenverteilung wahrgenommen wird. Daneben müssen Ideen vorhanden sein und artikuliert werden, die als legitime, machbare, passende, relevante und im Vergleich zu anderen Möglichkeiten als effektivere Lösung angesehen werden. Institutionelle Unternehmer*innen müssen vorhanden sein, die diese Lösung voranbringen und dafür über die nötigen Netzwerke verfügen.

Quelle: Campbell (2004). Eigene Darstellung 
(a) Politikwandel geschieht in Krisenzeiten, wenn durch Vorkommnisse von außen oder Widersprüche von innen Unsicherheiten vorhanden sind, welche die Akteur*innen zur Suche nach neuen Institutionen bewegen (Blyth 2002, S. 10 f.; Campbell 2004, S. 174 f.).

Einige Autor*innen argumentieren, dass in Zeiten von hoher Unsicherheit oder Krisen am ehesten institutioneller Wandel durch Ideen hervorgerufen wird. Dies können wirtschaftliche Katastrophen aber auch interne Widersprüche sein (Blyth 2002, S. 11, 270; Schmidt 2002, S. 225 f.). Krisen sind laut Hay (2006, S. 67) Momente, in denen Akteur*innen die Wahrnehmung ihrer Interessen in Frage stellen und in denen Kämpfe über die Definition der Krise und möglicher Lösungen stattfinden. In Krisenzeiten ist es für Akteur*innen schwierig oder unmöglich, mit ihren üblichen Programmen bestehende Probleme zu lösen (Campbell 2004, S. 115; Schmidt 2002, S. 225 f.). Auch ist den Entscheidungsträger*innen teilweise selbst unklar, was ihre Interessen sind (Campbell 2004, S. 115). In diesen Zeiten hoher Unsicherheit entsteht eine Offenheit gegenüber neuen Ideen und Werten, bestehende Werte und Ideen werden vermehrt herausgefordert. „It is only in those moments when uncertainty abounds and institutions fail that ideas have this truly transformative effect on interests" (Blyth 2002, S. 270). In diesen Phasen ermächtigen Ideen Akteur*innen dazu, bestehende Institutionen zu hinterfragen, reduzieren außerdem Unsicherheiten und ermöglichen Koalitionenbildung zwischen Anhänger*innen ähnlicher Ideen, ermöglichen die Konstruktion neuer Institutionen und koordinieren die Erwartungen der Akteur*innen (Blyth 2002, S. 15, 35). Doch auch interne Widersprüche können zu institutionellen Veränderungen führen, wenn beispielsweise kognitive und normative Argumente sich widersprechen (Schmidt 2002, S. 227) oder neue Machtkonstellationen entstehen:

\footnotetext{
„Veränderungen in den Interessen- und Machtkonstellationen, sozialer Wandel sowie neue Einstellungen und Präferenzen in einer Gesellschaft veranlassen individuelle und kollektive Akteure dazu, sich Institutionen immer wieder neu anzueignen, sie zu interpretieren und ,auszuhandeln': Dies sind die internen Triebfelder des Institutionenwandels.“ (Quack 2006, S. 180)
}

(b) Weitere Voraussetzung für Politikwandel ist, dass die Entscheidungsträger*innen die Situation als Krise und als Gefahr für die aktuelle Macht- und Ressourcenverteilung wahrnehmen (Blyth 2002, S. 9; Campbell 2004, S. 175 f.; King 1999, S. 39). 
Das Vorhandensein von Krisen alleine führt nicht automatisch zu Politikveränderungen, da eine weitere Voraussetzung ist, dass die Krisen von den Entscheidungsträger*innen als solche wahrgenommen werden und eine Suche nach neuen Lösungen angestoßen wird (Campbell 2004, S. 115-175).

Wird in Krisenzeiten das Versagen bestehender Politikinstrumente und Paradigmen deutlich und kann ein vorherrschendes Paradigma die Entwicklungen nicht mehr ausreichend erklären, stehen die Chancen besonders gut, dass ein neues Paradigma erfolgreich ein altes ersetzt (Hall 1993, S. 285). In diesen von Unsicherheit geprägten Zeiten wird in der Politik zunächst versucht, bestehende Instrumente oder das Paradigma geringfügig anzupassen oder das bestehende Problem umzudefinieren. Gelingt dies nicht, kann es zu einem Legitimationsverlust der Paradigmen und einem Paradigmenwechsel kommen (Hall 1993, S. 280; Hay 2001, S. 193).

Dabei werden Akteur*innen einen Wandel eher unterstützen, wenn sie annehmen, dass er ihre Ressourcen oder ihre Macht steigert, und eher versuchen ihn zu verhindern, wenn er ihre Ressourcen oder Macht voraussichtlich verringern wird (Campbell 2004, S. 176). Mit der Macht der Entscheidungsträger*innen ist hier ihre Möglichkeit gemeint, die Denk- und Handlungsalternativen bestimmter Gruppen einzuschränken, indem politische Entscheidungen getroffen oder indem Paradigmen und öffentliche Empfindungen beeinflusst werden (Knight 1992, S. 41 f.). Oder, bezogen auf drei von Carstensen und Schmidt (2016) beschriebene Formen von Macht, verfügen die Entscheidungsträger*innen insbesondere über ,power over ideas“ - die Möglichkeit, zu beeinflussen, welche Ideen diffundieren und politische Entscheidungen zu beeinflussen -, sowie ,power through ideas" - die Möglichkeit der bewussten Kommunikation von Ideen zur Beeinflussung des Denkens und Handelns anderer. Sehen die Entscheidungsträger*innen diese Macht gefährdet, unterstützen sie eher eine Idee für politische Veränderung, wenn sie annehmen, dass diese eine Stärkung ihrer Macht ermöglicht. Einmal von vielen Akteur*innen als gegeben angesehen, können jedoch auch Ideen selbst eine Macht entfalten, ,power in ideas“ (Carstensen und Schmidt 2016), sich gegenüber anderen Ideen durchsetzen und Veränderungen verhindern oder voranbringen. Pfadabhängigkeiten erschweren dabei aber Veränderungen, da der Aufbau neuer Institutionen stets aufwändiger ist als das Beibehalten des Status quo (Campbell 2004, S. 65-67).

(c) Eine neue Idee muss vorhanden sein und so kommuniziert werden, dass sie für das wahrgenommene Problem als im Vergleich zu anderen Ideen als effektivere Lösung gesehen wird und als legitim, angemessen und nützlich erscheint (Campbell 2004, S. 117, 179; Dacin et al. 2002, S. 47; Schmidt 2002, S. 217-219). 
Eine Idee, beispielsweise ein neues Programm, kann am ehesten dann durchgesetzt werden, wenn glaubwürdig gezeigt werden kann, dass es effektiver ist als ein anderes Programm in Bezug auf die wahrgenommene Krise. Sie muss wünschenswerter und nützlicher erscheinen als die bisherige und als andere neue Lösungsideen (Dacin et al. 2002, S. 47; vgl. auch Schmidt 2002, S. 217-219). Daneben müssen Entscheidungsträger*innen überzeugt werden, dass die Idee für den jeweiligen lokalen Kontext angemessen und legitim ist und dass sie sich einfach umsetzen lässt. Dazu ist es hilfreich, wenn die Idee bereits in anderen Kontexten erfolgreich umgesetzt wurde (Campbell 2004, S. 179). Hier spielen die in Abschnitt 3.2.3 genannten normativen und kognitiven Kriterien von Ideen in Diskursen eine Rolle, sowie Frames, die zur Legitimierung der Ideen genutzt werden.

(d) Institutionelle Unternehmer*innen mit finanziellen Ressourcen müssen vorhanden sein und ihre Idee einfach und verständlich formulieren (Campbell 2004, S. 177 f.; Schmidt 2002, S. 304).

Die Kommunikation der neuen Idee ist eine weitere notwendige Voraussetzung für Politikwandel. Dazu muss die Idee von den institutionellen Unternehmer*innen einfach und verständlich als geeignete Lösung für die bestehenden Probleme artikuliert werden. Bei der Ablösung von Paradigmen gehören, zumindest in demokratischen Gesellschaften, auch Diskussionen der Paradigmen in der Öffentlichkeit dazu, beispielsweise in den Medien (Campbell 2004, S. 34; Hall 1993, S. 286; Hay 2001, S. 200). Dafür sind personelle und auch weitere Ressourcen notwendig, weshalb institutionelle Unternehmer*innen größere Chancen haben, ihre Ideen durchzusetzen, wenn sie über mehr finanzielle Ressourcen verfügen als die Verfechter*innen konkurrierender Ideen (Campbell 2004, S. 178 f.).

(e) Institutionelle Unternehmer*innen müssen vernetzt sein, insbesondere zu den Entscheidungsträger*innen und müssen die Idee an diese kommunizieren (Campbell 2004, S. 178 f.).

Um ihre Idee zu verbreiten und zur Umsetzung zu bringen, müssen die institutionellen Unternehmer*innen Zugang zu den jeweils relevanten Entscheidungsträger*innen haben und ihnen die Idee vermitteln. Daneben benötigen die institutionellen Unternehmer*innen Unterstützung von anderen Akteur*innen, weshalb sie ihre Idee in unterschiedlichen Kontexten verbreiten müssen (Campbell 2004, S. 178 f.).

Es lässt sich also festhalten, dass das Vorhandensein von Krisen, die als solche anerkannt und kommuniziert werden, notwendige Voraussetzung für Politikwandel ist. Darüber hinaus werden alternative Ideen für neue politische Lösungen 
benötigt sowie institutionelle Unternehmer*innen, die diese kommunizieren (siehe Abb. 3.2). Daneben gibt es eine Reihe von Kriterien dafür, ob der Wandel eher graduell oder transformativ ausfällt, die in den folgenden Thesen dargestellt werden und in Tabelle 3.2 aufgeführt sind.

Tab.3.2 Voraussetzung für graduellen und transformativen Wandel

\begin{tabular}{l|l}
\hline Gradueller Wandel & Transformativer Wandel \\
\hline $\begin{array}{l}\text { Ideen, die besser in den bestehenden } \\
\text { Kontext passen }\end{array}$ & $\begin{array}{l}\text { Ideen, die kaum in den Kontext passen und } \\
\text { mehr Veränderung erfordern }\end{array}$ \\
\hline $\begin{array}{l}\text { Institutionelle Unternehmer*innen sind } \\
\text { weniger vernetzt }\end{array}$ & $\begin{array}{l}\text { Institutionelle Unternehmer*innen haben } \\
\text { größere Netzwerke }\end{array}$ \\
\hline $\begin{array}{l}\text { Entscheidungsträger*innen sind nicht } \\
\text { vollkommen von der Idee überzeugt und } \\
\text { stellen nicht die nötigen Ressourcen bereit }\end{array}$ & $\begin{array}{l}\text { Entscheidungsträger*innen stehen voll } \\
\text { hinter der Idee und nutzen die notwendigen } \\
\text { Ressourcen }\end{array}$ \\
\hline
\end{tabular}

Die Tabelle zeigt Kriterien dafür, wann ein Politikwandel eher graduell, als Wandel erster oder zweiter Ordnung, und wann eher transformativ (Wandel dritter Ordnung) verläuft. Wie der Tabelle zu entnehmen ist, hängt dies von der Passung der Ideen, den Netzwerken der institutionellen Unternehmer*innen sowie den Einstellungen der Entscheidungsträger*innen $a b$.

Quelle: Campbell (2004). Eigene Darstellung.

(f) Ideen, die besser in den bestehenden Kontext passen, können zwar einfacher umgesetzt werden, bewirken dann aber eher einen graduellen als einen transformativen Wandel (Campbell 2004, S. 179-181).

Ideen, die in bestehende institutionelle Kontexte passen, können sich leichter durchsetzen ohne abgewandelt oder verworfen $\mathrm{zu}$ werden als Ideen, die große Veränderungen erfordern. Dies betrifft sowohl die Übereinstimmung mit bestehenden Gesetzen als auch mit Paradigmen oder Empfindungen der Öffentlichkeit. Ideen, die besonders gut in den bestehenden Kontext passen - also Policies oder Programme, die mit bestehenden Paradigmen und Empfindungen übereinstimmen - sorgen folglich für einen Wandel eher geringeren Grades und hinterfragen nicht die im Hintergrund stehenden Ideen (Campbell 2004, S. 179-181).

(g) Institutionelle Unternehmer*innen, die verschiedenen Netzwerken angehören und dadurch Zugang zu einem breiteren Ideenspektrum haben, können eher radikale Ideen durchsetzen als Akteur*innen die weniger breit vernetzt sind (Campbell 2004, S. 178).

Neue Ideen sind in den meisten Fällen eine innovative Kombination aus verschiedenen vorhandenen Ideen. ,[...] [A]ctors often craft new institutional solutions 
by recombining elements in their repertoire through an innovative process of $\mathrm{bri}$ colage whereby new institutions differ from but resemble old ones" (Campbell 2004, S. 69). Mit bricolage meint Campbell ebendiese Zusammenstellung vorhandener Ideen verschiedener Kontexte zu einer neuen innovativen Idee. Daher fällt der Wandel umso geringer aus, je näher die neue Idee an den bisher bestehenden ist und umso eher ist der Wandel von Pfadabhängigkeiten gekennzeichnet. Grundlegende Annahmen werden dann nicht in Frage gestellt.

Sind institutionelle Unternehmer*innen in verschiedenen Netzwerken verankert und haben aus diesem Grund Zugang zu einem breiten Ideenspektrum, so können sie radikalere neue Ideen formulieren. Sind sie weniger breit vernetzt, so bewirkt ihr Zugang zu einem nur sehr begrenzten Repertoire an Ideen das Entstehen weniger radikaler Ideen. Dementsprechend fällt der angestoßene Wandel dann eher graduell als transformativ aus (Campbell 2004, S. 178).

(h) Politikwandel kann eher dann einen höheren Grad erreichen, wenn die Entscheidungsträger*innen die neue Idee unterstützen und die nötigen Ressourcen für die Umsetzung einsetzen (Campbell 2004, S. 181).

Da die Veränderung von Paradigmen oder gar Institutionen mehr Aufwand bedeutet als ein Wandel erster oder zweiter Ordnung, werden Entscheidungsträger*innen stets erst versuchen, nur Policies und Programme zu verändern, es sei denn sie sind von der Notwendigkeit der neuen Paradigmen überzeugt. Dafür müssen sie zu der Einschätzung gelangen, dass sie über die dafür notwendigen Ressourcen verfügen und diese einsetzen können (Campbell 2004, S. 181). Daher ist ein transformativer Wandel wahrscheinlicher, wenn die Entscheidungsträger*innen die Idee unterstützen und ihre Ressourcen für dessen Umsetzung zur Verfügung stellen.

\subsection{Bisherige Anwendungen des diskursiven Institutionalismus}

Der theoretische Ansatz des diskursiven Institutionalismus und die sich daraus ergebenden Forschungsfragen und methodischen Vorgehen wurden in den vergangenen Jahren von zahlreichen Autor*innen verwendet. Nachdem in den vorangegangenen Abschnitten die Grundgedanken, Konzepte und Annahmen der Theorie dargestellt wurden, wird im Folgenden nun gezeigt, in welchen Themenfeldern und mit welchen Methoden der Theorieansatz bisher Anwendung fand. Daraus können möglicherweise Schlüsse gezogen werden, für welche Anwendungen sich die Theorie eignet, welche Methoden in Frage kommen und welche Fragen bisher offengeblieben sind. 
In den meisten Studien ist bereits zu Beginn der Analyse deutlich, ob es zu einer Veränderung kam oder nicht; die Autor*innen analysieren dann die Ursache und den genauen Prozess der Veränderung. Bei einem Blick auf Studien, die den diskursiven Institutionalismus als Grundlage verwenden, fällt außerdem auf, dass die meisten Studien mit einzelnen Fallstudien die nationale Ebene untersuchen oder Diskurse und politische Entscheidungen im Ländervergleich untersuchen (u. a. Berman 1998; Boswell und Hampshire 2017; Di Gregorio et al. 2017; Kern 2011; Lieberman 2007; Ochieng et al. 2016; Schmidt 2002, 2014; K. Smith 2013; Wallaschek 2020). Nur wenige Studien untersuchen Diskurse und Ideen auf lokaler Ebene (u. a. Gardner 2017; Granqvist et al. 2020; Romsdahl et al. 2017). So beziehen sich auch die meisten Hypothesen zur Bedeutung von unterschiedlichen Arten von Diskursen und Ideen auf die nationale Ebene der Politik.

Besonders häufig wurde der diskursive Institutionalismus außerdem im Zusammenhang mit Studien zur Europäisierung oder Politik der Europäischen Union verwendet (u. a. Fairbrass 2011; Heidbreder 2013; Heron und Murray-Evans 2016; Herranz-Surralles 2016; Lauber und Schenner 2011; Nordin 2017; Rayroux 2014; Risse 2001; Schmidt 2006, 2014, 2016; Wallaschek 2020; Wendler 2019). Fairbrass (2011) findet in ihrer Studie heraus, dass die Diskussionen um Corporate Social Responsibility auf europäischer Ebene hauptsächlich in koordinativen Diskursen unter Einbeziehung nur weniger Akteur*innen stattgefunden haben.

Darüber hinaus wenden Autor*innen den diskursiven Institutionalismus im Zusammenhang mit unterschiedlichen Entscheidungen und Entwicklungen in wirtschafts- und finanzpolitischen Fragen an (u. a. Bakir 2009; Campbell-Verduyn 2017; Heron und Murray-Evans 2016; Hope und Raudla 2012; Schmidt 2002, 2016; Warren 2020; Widmaier 2016) und mit der Analyse von Veränderungen der sozialdemokratischen Parteien in Deutschland und Schweden (Berman 1998). Bei einem Vergleich der Steuerpolitik in Estland und den USA kommen Hope und Raudla (2012) zu dem Schluss, dass der diskursive Institutionalismus auch zur Erklärung von politischer Stabilität geeignet ist und auch der Mangel an Veränderung ein Ergebnis von Diskursaktivitäten sein kann. Andere Analysen, für welche die Autor*innen den diskursiven Institutionalismus verwenden, beschäftigen sich unter anderem mit nationalen und europäischen Identitäten (Risse 2001), den politischen Entscheidungen für oder gegen die Einführung der Ehe zwischen gleichgeschlechtlichen Paaren (Grube und van Acker 2017) oder der Gesundheitspolitik (K. Smith 2013). Vermehrt wird diese Theorieströmung auch für Studien im Nachhaltigkeitsbereich verwendet, wie zur Untersuchung von Umwelt-, Energie- und Klimapolitik (u. a. Buijs et al. 2014; den Besten et al. 2014; Di Gregorio et al. 2017; Gillard 2016; Henrysson und Hendrickson 2020; Kern 2011; Klenk und Larson 2015; Ochieng et al. 2016; Romsdahl et al. 2017; 
Vijge 2013; Wendler 2019). In ihrer Studie kommen Romsdahl et al. (2017) zu dem Schluss, dass in unterschiedlichen Städten sich die Narrative und Diskurse über den Klimawandel durchaus unterscheiden, um besser an lokale Begebenheiten anzuschließen. Diese Diskursunterschiede können dann auch unterschiedliche politische Entscheidungen mit sich bringen.

Die meisten Studien verwenden als Methode zur Analyse der Ideen und Diskurse qualitative Interviews (u. a. Bosomworth 2018; Gardner 2017; Granqvist et al. 2020; Kern 2011; Kromidha und Cordoba-Pachon 2017; Lauber und Schenner 2011; Romsdahl et al. 2017), Online-Fragebögen (u. a. Bosomworth 2018; Romsdahl et al. 2017) und Dokumentenanalysen, beispielsweise von Zeitungsartikeln oder Politikdokumenten (u. a. Fairbrass 2011; Granqvist et al. 2020; Henrysson und Hendrickson 2020; Kern 2011; Kromidha und Cordoba-Pachon 2017; Lauber und Schenner 2011; Romsdahl et al. 2017; Stassen et al. 2010).

Boswell und Hampshire (2017) untersuchen in ihrer Studie verschiedene Strategien, die politische Akteur*innen nutzen, um ausgewählte Ideen gegenüber anderen hervorzuheben. Eine dieser Strategien ist das Betonen der technischen Seite einer Idee, wodurch mögliche Widersprüche zu verbreiteten normativen Ideen nicht deutlich werden und Diskussionen auf grundlegender Ebene möglichst vermieden werden können. Heidbreder (2013) zeigt am Beispiel der EU, wie öffentliche Empfindungen von Paradigmen entkoppelt werden, wenn sie sich widersprechen. So existieren die Werte und Ziele nationaler Souveränität parallel mit dem nach europäischer Integration, sowie Politikinstrumente für beide Seiten, gleichwohl die im Hintergrund liegenden Ideen sich widersprechen. Bei der Legitimierung der Ideen kann es in einigen Fällen jedoch zu Schwierigkeiten kommen, wenn Politikinstrumente offensichtlich nicht zu den öffentlichen Empfindungen passen.

Kern (2011) dagegen untersucht anhand der Energiepolitik in den Niederlanden und Großbritannien, wie Interessen, Diskurse und Institutionen zusammenhängen. Er kommt dabei zu dem Ergebnis, dass radikaler Wandel dann vorkommen kann, wenn die neuen Diskurse sowohl den bestehenden Interessen der im jeweiligen Feld wichtigen politischen Akteur*innen widersprechen, als auch bestehende Institutionen herausfordern. Ansonsten seien nur kleinere oder keine Veränderungen zu erwarten. Oft würden aber im Prozess der Institutionalisierung die neuen Ideen insoweit abgeschwächt, dass sie die bestehenden Interessen oder Institutionen unterstützen. Kern liefert allerdings keinen Ansatzpunkt, wann und mit welchen diskursiven Strategien diese erfolgreiche Durchsetzung von neuen Diskursen ohne Anpassung an bestehende Interessen und Institutionen erfolgreich ist. Wichtig für die vorliegende Arbeit ist jedoch seine Schlussfolgerung, dass durch unterschiedliche Interessen und Institutionen in verschiedenen Kontexten keine universelle Lösung für erfolgreiche Energietransformation möglich 
ist, da aufgrund der bestehenden Rahmenbedingungen jeweils unterschiedlich auf Lösungsvorschläge reagiert wird (Kern 2011, S. 1130). Dieses Forschungsergebnis kann womöglich auch auf andere Bereiche von Transformation übertragen werden, wo auch stets die Rahmenbedingungen einer gewünschten Veränderung beachtet werden müssen, was in der vorliegenden Arbeit vorgenommen wird.

Bezüglich der Operationalisierung von Wandel erster, zweiter und dritter Ordnung schlägt Gardner (2017, S. 154 f.) vor, Wandel erster und zweiter Ordnung als materiellen Wandel zu begreifen, während Wandel dritter Ordnung diskursiv erfolgt. Dies widerspricht jedoch dem Argument von Hall (1993, S. 179, Endnote 21), Wandel dritter Ordnung würde zusätzlich die Veränderungen der Instrumente (zweite Ordnung) und deren Ausgestaltung (erste Ordnung) mit sich bringen. Umgekehrt beinhalten auch Veränderungen erster und zweiter Ordnung diskursive Veränderungen, da dort ebenfalls Ideen ausgetauscht und durchgesetzt werden.

\subsection{Zwischenfazit: Zusammenführung der theoretischen Konzepte und Schlussfolgerungen für die Analyse}

Der diskursive Institutionalismus ist im Vergleich zu den anderen Strömungen des Neoinstitutionalismus dadurch gekennzeichnet, dass der Fokus auf der Rolle von Ideen bei Politikveränderungen liegt. Gleichzeitig beinhaltet der diskursive Institutionalismus aber auch einige Argumente der anderen Strömungen, indem Pfadabhängigkeiten ebenfalls als einschränkend für Politikwandel wahrgenommen werden, die Interessen von Entscheidungsträger*innen und ihr Streben nach Macht und Ressourcen auch eine Rolle spielen ebenso wie ihre Werte, Normen und Identitäten. Der Fokus liegt bei dem diskursiven Institutionalismus jedoch auf der Kommunikation von Ideen durch Akteur*innen in Diskursen. Es wird angenommen, dass Politikveränderungen nicht nur durch exogene Ereignisse ermöglicht werden, sondern dass auch durch interne Widersprüche und Krisen neue Ideen an Bedeutung gewinnen und in der Folge Politikwandel mit sich bringen können.

Dieser Wandel kann unterschiedliche Arten von Ideen betreffen. So werden hier, angelehnt an die drei Ebenen von Ideen nach Schmidt (2008) sowie die Kategorisierung von Campbell (2004), fünf Arten von Ideen unterschieden: Policies, Programme, Frames, Paradigmen und öffentliche Empfindungen. Im Vordergrund, in Diskursen explizit benannt und den Akteur*innen bewusst, sind die Policies, Programme und Frames. Paradigmen und öffentliche Empfindungen dagegen liegen im Hintergrund der Diskurse, werden oftmals nicht explizit formuliert und 
sind vielen Akteur*innen nicht bewusst. Während sich Policies und Frames häufig und leicht verändern, ist dies bei Programmen etwas schwieriger. Paradigmen und öffentliche Empfindungen lassen sich nur sehr schwer und selten verändern. Wenn dies aber geschieht, dann stellt es einen Wandel höherer Intensität dar, als lediglich die Einführung einer neuen Policy.

Daran schließt die Unterscheidung von drei Graden der Veränderung nach Hall (1993) an, die hier daher mit der Kategorisierung von Ideen nach Schmidt (2008) und Campbell (2004) in Verbindung gebracht wird (siehe Abb. 3.3). Wenn sich lediglich Policies oder Frames verändern, führt dies zu einem Wandel erster Ordnung. Werden umfassende Programme neu eingeführt, kommt es zu einem Wandel zweiter Ordnung. Beide Formen stellen einen graduellen Wandel dar. Verändern sich dagegen Paradigmen und öffentliche Empfindungen und damit einhergehend nach und nach auch die daraus abgeleiteten Policies, Programme und Frames, so kommt es zu einem Wandel dritter Ordnung. Dieser wird auch als Transformation bezeichnet. In allen drei Fällen kann es zu einer Institutionalisierung der neuen Ideen kommen, was aber nicht immer der Fall ist.

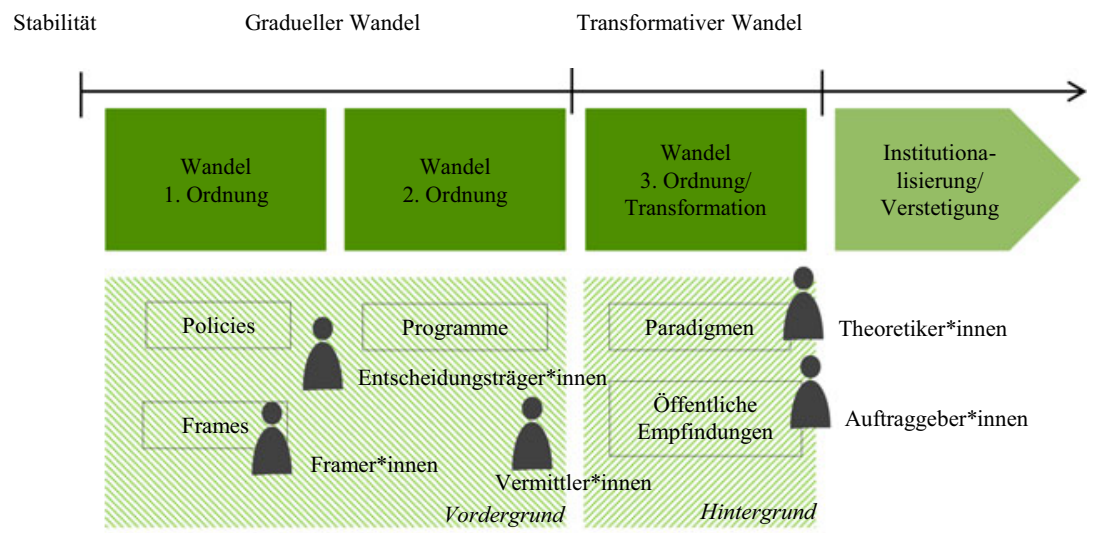

Schwierigkeitsgrad der Veränderung

Abb. 3.3 Ideen, Akteur*innen und Wandel im diskursiven Institutionalismus. Die Abbildung zeigt den Zusammenhang zwischen verschiedenen Kategorien von Ideen und Gruppen von Akteur*innen, die diese voranbringen. Außerdem werden die Ideen den unterschiedlichen Graden von Politikwandel zugeordnet, wobei die Institutionalisierung separat betrachtet werden muss, da aus allen Ideen und Graden von Wandel Institutionalisierung folgen kann. Quelle: Schmidt (2008), Campbell (2004), Hall (1993), Hay (2001). Eigene Darstellung. 
Während von einigen Autor*innen auch Paradigmen als Institutionen verstanden werden (Senge und Hellmann 2006, S. 18), werden hier, um die Unterscheidung zwischen Institutionen als formelle Regeln und Organisationen und den dahinterliegenden Sinnstrukturen aufrecht zu erhalten, Institutionen enger definiert (Hay 2001, S. 213), und zwar als formelle Regeln, Gesetze und Organisationsstrukturen. Statt von institutionellem Wandel wird hier daher allgemeiner von Politikwandel gesprochen, mit dem eine Veränderung der vorherrschenden Ideen (Paradigmen, Empfindungen, Frames, Programme und Policies) sowie in dessen Folge der in diesem Zusammenhang stehenden Institutionen gemeint ist. In der folgenden Analyse sind einerseits das Aufkommen und die Diffusion von neuen Ideen sowie daraus möglicherweise folgende Veränderungen von Politik von Interesse. Sind diese tiefgreifend auf der Ebene von Paradigmen angesiedelt, stellen sie eine Transformation dar. Werden die Paradigmen anschließend institutionalisiert, können sie längerfristig verstetigt werden. Andererseits werden hier auch institutionelle Veränderungen im Kontext der Fallbeispiele betrachtet, die jedoch im Sinne von veränderten Rahmenbedingungen betrachtet werden, innerhalb derer die institutionellen Unternehmer*innen agieren.

$\mathrm{Ob}$ und zu welchem Grad von Veränderung es kommt - ob also eine Transformation erfolgt oder lediglich Veränderungen erster oder zweiter Ordnung - hängt von der Art der vorgeschlagenen Lösungen, deren Passung mit dem bestehenden Kontext, der Art, wie die Lösungen im Kontext kommuniziert werden sowie zusätzlich von den Interessen, der Macht und den Ressourcen der beteiligten Akteur*innen ab (siehe Abschn. 3.3.2). Hierbei wird deutlich, wie der diskursive Institutionalismus in seinem Erklärungsansatz Aspekte aus verschiedenen Strömungen des Neoinstitutionalismus zusammenbringt. So liegt der Fokus auf Akteur*innen mit ihren Ideen und ihrer Kommunikation in Diskursen; die Rolle von Interessen, Ressourcen und Kontextfaktoren wird jedoch auch berücksichtigt. Dabei ist das Vorhandensein und die Kommunikation einer Krise, die von den Entscheidungsträger*innen als Gefahr für ihre Macht- und Ressourcenausstattung wahrgenommen wird, Voraussetzung für Politikwandel. Weiterhin muss eine neue Idee als alternative Lösung vorhanden sein und so kommuniziert werden, dass sie als geeignet, legitim, passend und machbar angesehen wird und einer anderen Idee vorgezogen wird. Dies geschieht, indem institutionelle Unternehmer*innen diese Idee voranbringen und dabei auf gute Netzwerke und den Zugang zu Entscheidungsträger*innen zurückgreifen können. Während Ideen, die besser in den Kontext passen, leichter umgesetzt werden können und einen graduellen Wandel mit sich bringen, können radikalere und weniger passende Ideen zwar schwieriger umgesetzt werden, bewirken, wenn sie erfolgreich sind, dann aber eher eine Transformation. 
Den in der Theorie genannten Arten von Ideen können Gruppen von Akteur*innen zugeordnet werden, die diese in Diskursen voranbringen (siehe Abschn. 3.2.2). Nach der Unterscheidung von Campbell (2004, S. 100-107) bringen Entscheidungsträger*innen insbesondere Policies und Programme voran. Framer*innen helfen dabei, diese passend für den Kontext und die öffentlichen Empfindungen zu formulieren. Theoretiker*innen entwickeln Paradigmen, verbreiten diese und leiten daraus Programme und Policies her. Die Auftraggeber*innen sind als Zielgruppe der Programme und Policies von ihren Empfindungen, also Werten, Normen und Identitäten, beeinflusst und bewerten, ob eine vorgeschlagene Idee dazu passt. Zwischen diesen Akteur*innen stehen Vermittler*innen, die die Ideen von einem Diskurs in einen anderen und zwischen den Gruppen von Akteur*innen verbreiten und für den jeweiligen Kontext passend übersetzen. Die institutionellen Unternehmer*innen, die neue Ideen voranbringen und damit einen Politikwandel intendieren, können aus den unterschiedlichen Gruppen von Akteur*innen kommen (Campbell 2004, S. 178). Aufgrund der Verortung der Paradigmen bei den Theoretiker*innen stellt sich die Frage, ob ein Wandel dritter Ordnung nur möglich oder zumindest wahrscheinlicher ist, wenn die institutionellen Unternehmer*innen aus der Gruppe der Theoretiker*innen stammen oder ob auch andere Akteur*innen neue Paradigmen voranbringen und Transformation anstoßen können.

Eben diese Transformation wird bei der transformativen Forschung angestrebt, bei der Forschende selbst als institutionelle Unternehmer*innen - hier als Pionier*innen des Wandels bezeichnet - agieren, neue Ideen entwickeln und in politische Prozesse einbringen (siehe Abschn. 2.2.2). Da sie dies in den meisten Fällen auf der Ebene von Städten versuchen, der diskursive Institutionalismus jedoch bisher kaum die lokale Ebene betrachtet, stellt sich die Frage, ob Veränderungen dritter Ordnung, wie vom diskursiven Institutionalismus verstanden, in dem begrenzten Raum von Städten überhaupt möglich sind. Aufgrund der Überschneidung der Definitionen von Eigenlogik als ,strukturierte und strukturierende Handlungen“ (Löw 2008, S. 77) und Paradigmen sowie der Beobachtung von durchaus unterschiedlichen Krisendefinitionen, Lösungsstrategien und Diskursen in Städten (u. a. Barbehön et al. 2015; Barbehön und Münch 2017; Romsdahl et al. 2017) wird in der vorliegenden Arbeit davon ausgegangen, dass sich durchaus in Städten eigenständig neue Paradigmen entwickeln und verbreiten können (siehe Abschn. 2.3).

Auch die Transition-Forschung sowie das teilweise daran angelehnte Transformationsverständnis des WBGU (2011), der die transformative Forschungsperspektive vorgestellt hat, sehen in Städten durchaus die Möglichkeit einer 
umfassenden Transformation. Insgesamt zeigen diese drei Ansätze viele Ähnlichkeiten in ihrem Verständnis von Transformationsprozessen, wie deren Voraussetzungen, Dauer, Umfang sowie die Art der Veränderung (siehe Tab. 3.3). Während die Transition-Forschung und der WBGU lediglich Transformationen betrachten, unterscheidet und untersucht der diskursive Institutionalismus drei Grade von Veränderungen, von denen Transformation die umfassendste ist. Alle drei Ansätze verstehen Transformation als einen umfassenden, systemübergreifenden, fundamentalen Wandel, der einen Paradigmenwechsel beinhaltet, wobei eine Teilströmung der sich auf die MLP beziehenden Transition-Forschung auch das Ersetzen einzelner Technologien als Transformation bezeichnet. In Bezug auf die zeitliche Dauer der Veränderung kann diese über mehrere Jahrzehnte geschehen, wobei sowohl der WBGU (2011) als auch einige Autor*innen aus dem Kontext der MLP (u. a. Park et al. 2012) den eigentlichen Umbruch als weniger als zehn Jahre andauernd ansehen, dem ein längerer Prozess der Destabilisierung vorangegangen ist. Während der diskursive Institutionalismus sich allgemein mit Transformationen beschäftigt und diese als anhand von alternativen Paradigmen ausgerichtet ansieht, betrachten die Transition-Forschung sowie die transformative Forschung vor allem die von ihnen geforderte Nachhaltigkeitstransformation. Auch diese spezielle Form der Transformation ist in Richtung einer vorhandenen Vision einer nachhaltigen Gesellschaft ausgerichtet.

Größere Unterschiede der Betrachtungsweise, wenngleich nicht unbedingt Widersprüche, zeigen sich bezüglich des Kontextes, der Ursachen sowie der Prozesse der Transformation. So sehen zwar alle drei Perspektiven die Rolle von Akteur*innen mit neuen Ideen oder Technologien als zentral an. Der diskursive Institutionalismus beschreibt die Rolle der institutionellen Unternehmer*innen in allen Phasen der Transformation sowie ihre notwendigen Aktivitäten und Eigenschaften (siehe Abschn. 3.3.2). Während die MLP insbesondere die Diffusion der Innovationen in die Regime herein betrachtet, wenn Druck von außen die vorherrschenden Strukturen destabilisiert, spielen Nischenakteur*innen laut WBGU (2011) insbesondere am Anfang einer Transformation eine besondere Rolle, wenn neue Werthaltungen verbreitet und Alternativen aufgezeigt werden (siehe Abschn. 2.5). Der WBGU (2011) weist zusätzlich noch darauf hin, dass diese Ereignisse und Druck von außen nicht nur Wandel erleichtern, sondern im Gegenteil auch erschweren können. Auch der diskursive Institutionalismus betont die Rolle von äußeren Ereignissen und Problemen, differenziert hier jedoch noch weiter aus, dass Krisen von den Entscheidungsträger*innen wahrgenommen und kommuniziert werden müssen, was ein langer Prozess sein kann.

In allen drei Erklärungsansätzen spielen Pfadabhängigkeiten eine große Rolle, die Veränderungen hemmen. Alle drei Perspektiven nennen weitere förderliche 


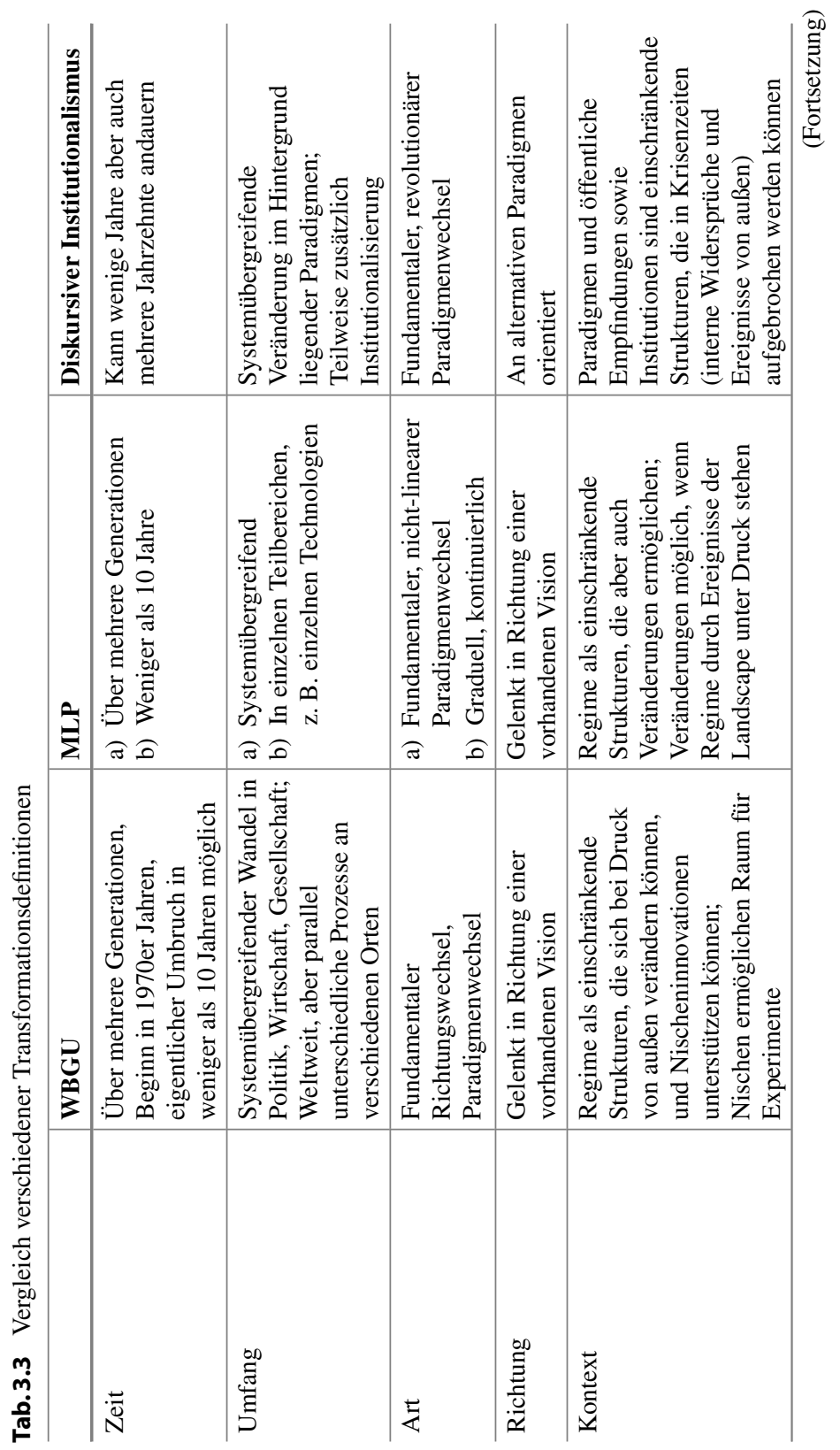




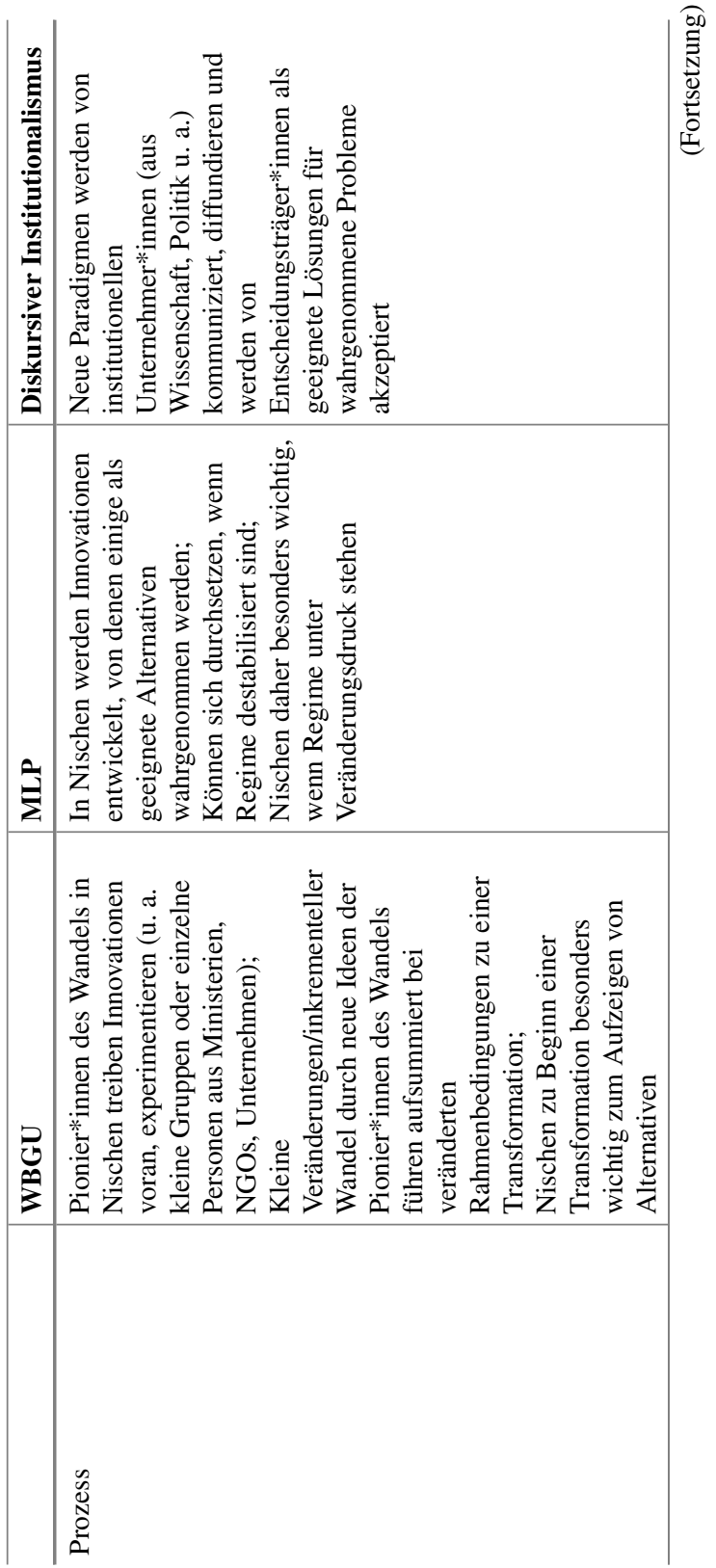




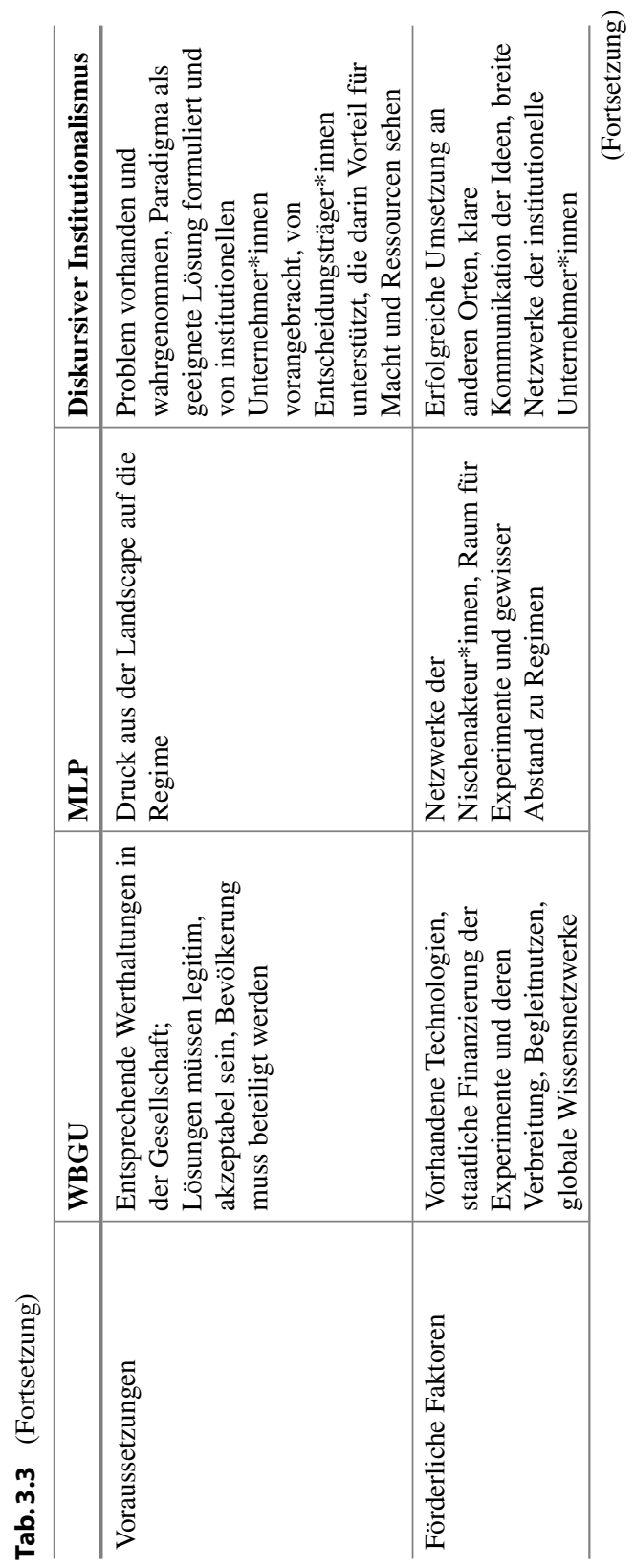




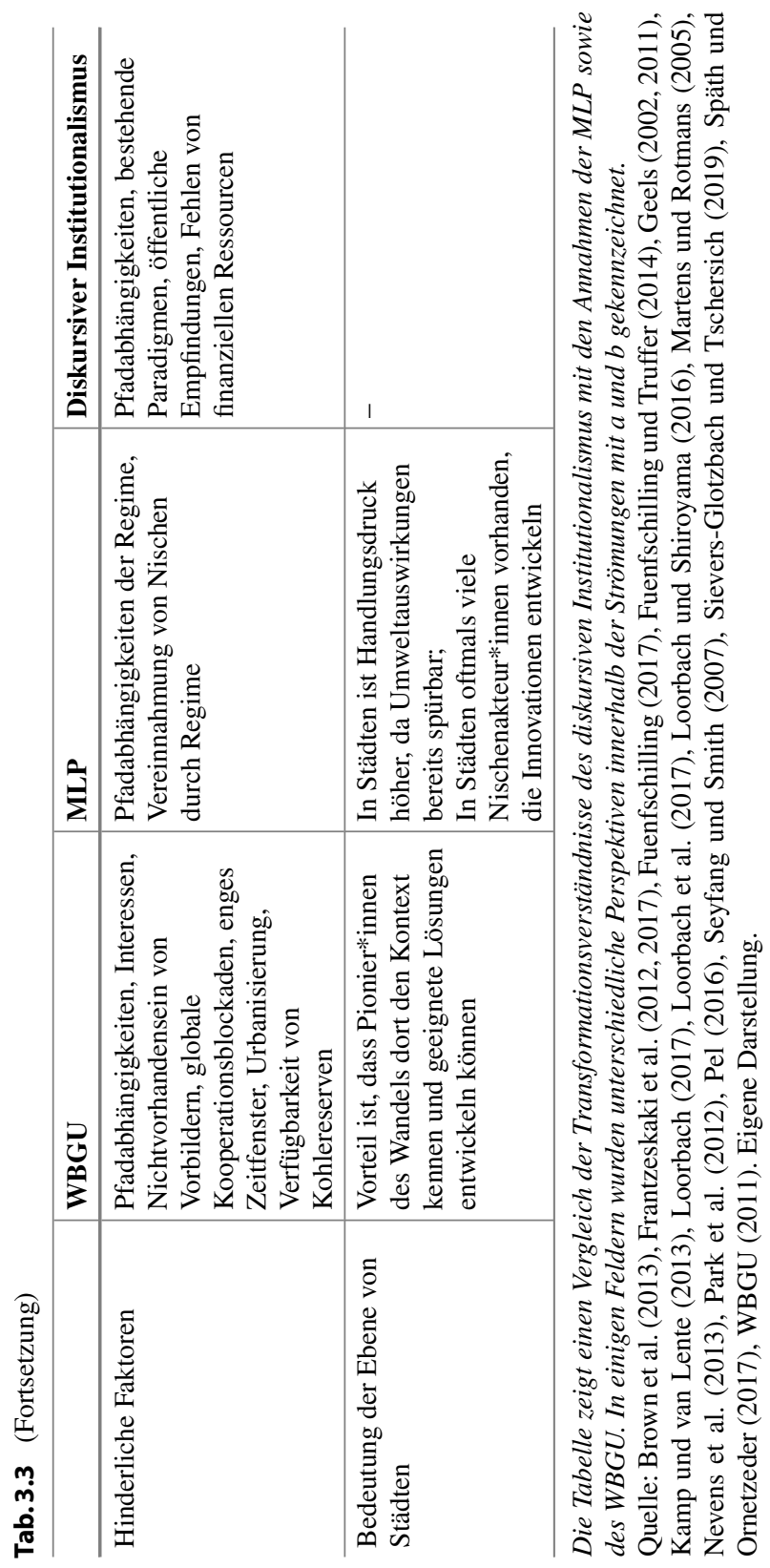


und hinderliche Faktoren für eine erfolgreiche Transformation (siehe Tab. 3.3), wobei der diskursive Institutionalismus dabei bereits tiefgreifender entwickelt ist als die anderen Perspektiven und konkrete Thesen nennt (siehe Abschn. 3.3.2), wohingegen bei der MLP und den Ausführungen des WBGU in seinem Gutachten von 2011 und anderer Autor*innen im Zusammenhang mit der transformativen Forschung einige Punkte unklar bleiben. So meint der WBGU (2011), dass verschiedene kleine Maßnahmen und Experimente der Pionier*innen des Wandels inkrementelle Veränderungen bewirken, die gemeinsam für eine Dynamik sorgen, welche unter veränderten Rahmenbedingungen vonseiten der Politik eine Transformation ermöglichen. Wie genau diese kleinteiligen Veränderungen zu einer Transformation - einem fundamentalen Paradigmenwechsel - führen, bleibt weitestgehend unklar. Die MLP, auf die sich die transformative Forschung stützt, widmet den veränderten Paradigmen nicht ausreichend Aufmerksamkeit, um diese Frage abschließend zu beantworten. Auch wird bei der MLP die Rolle der Entscheidungsträger*innen kaum beleuchtet und nicht genauer ausdifferenziert, welche Eigenschaften Nischeninnovationen erfüllen und welche Strategien Nischenakteur*innen anwenden müssen, um ihre Innovationen in die Regime zu überführen, wann also aus einer Diffusion eine Transformation folgt. An dieser Stelle könnte der diskursive Institutionalismus möglicherweise hilfreiche Beiträge leisten, um die von Forschenden angestoßenen Veränderungen in Richtung alternativer Paradigmen untersuchen zu können. Der diskursive Institutionalismus ist dagegen bisher nicht systematisch auf die lokale Ebene - beispielsweise eine Stadt - angewendet worden, wodurch weitestgehend offen bleibt, wie die Prozesse einer Transformation auf der lokalen Ebene konkret ablaufen, wobei die Perspektiven der MLP und der transformativen Forschung Ergänzungen liefern könnten.

Dadurch wird die Theorie des diskursiven Institutionalismus mit ihren in den vergangenen Abschnitten genannten zentralen Thesen weiterentwickelt und gleichzeitig für die transformative Forschung ein theoretisch fundiertes Analysetool entwickelt. Einerseits dienen die hier vorgestellten Transformationsdefinitionen der MLP sowie der transformativen Forschung zur Einordnung des Untersuchungsgegenstandes der transformativen Forschung. Die vorliegende Arbeit stützt sich auf das beschriebene Transformationsverständnis des diskursiven Institutionalismus. Da der WBGU sowie die MLP sich jedoch auch auf sehr ähnliche Definitionen beziehen, kann die transformative Forschung als Untersuchungsgegenstand gut in das Konzept des diskursiven Institutionalismus eingebettet und anhand daraus abgeleiteter Kriterien untersucht werden. Andererseits können daraus möglicherweise im Laufe der Analyse Thesen herausgearbeitet werden, die zur Weiterentwicklung des diskursiven Institutionalismus und zur spezifischen Anwendung auf der lokalen Ebene dienen. 
Open Access Dieses Kapitel wird unter der Creative Commons Namensnennung 4.0 International Lizenz (http://creativecommons.org/licenses/by/4.0/deed.de) veröffentlicht, welche die Nutzung, Vervielfältigung, Bearbeitung, Verbreitung und Wiedergabe in jeglichem Medium und Format erlaubt, sofern Sie den/die ursprünglichen Autor(en) und die Quelle ordnungsgemäß nennen, einen Link zur Creative Commons Lizenz beifügen und angeben, ob Änderungen vorgenommen wurden.

Die in diesem Kapitel enthaltenen Bilder und sonstiges Drittmaterial unterliegen ebenfalls der genannten Creative Commons Lizenz, sofern sich aus der Abbildungslegende nichts anderes ergibt. Sofern das betreffende Material nicht unter der genannten Creative Commons Lizenz steht und die betreffende Handlung nicht nach gesetzlichen Vorschriften erlaubt ist, ist für die oben aufgeführten Weiterverwendungen des Materials die Einwilligung des jeweiligen Rechteinhabers einzuholen. 